\title{
INFLUENCE OF POLAR SURFACE FORCES ON MAGNETIC FLOCCULATION OF FINE PARAMAGNETIC MINERALS. II. EXPERIMENT.
}

\author{
JIŘ́ ŠKVARLA \\ Department of Mineral Processing and Environmental Protection, Technical \\ University, 04384 Košice, Czechoslovakia
}

(Received August 29, 1991)

Abstract A model of interaction of colloids, taking into account the polar surface forces, has been applied to magnetic flocculation of siderite suspension in the presence of sodium oleate and sodium dodecylsulphate.

\section{INTRODUCTION}

In the previous theoretical study [1] a model of interaction between fine minerals in external homogeneous magnetic field was developed by incorporating the effect of polar surface forces. These forces are a manifestation of the so-called polar interfacial interactions between interacting solid surfaces and surrounding polar liquid. It was shown that these interactions will play an important role in magnetic flocculation of fine paramagnetic minerals.

The purpose of the present study is the determination of the aggregation behaviour of a fine paramagnetic mineral due to magnetic dipolar and polarstructural forces and the correlation of these results with theoretical conclusions of the model presented earlier [1].

In an attempt to match the conditions of the theory and to vary wettability (which is related to the polar interfacial interactions) of the mineral in a wide range, the aggregation tests have been conducted on siderite suspensions in the presence of sodium oleate and sodium dodecylsulphate making siderite hydrophobic. 


\section{EXPERIMENTAL}

Fine siderite $(-5 \mu \mathrm{m})$ from Rudnany (Czechoslovakia) was used in all investigations.

To assess the degree of aggregation time, changes in optical turbidity of the suspension, that accompany the sedimentation, were recorded photoelectrically.

A special cassette that was able to measure particular voltage changes for small quantities of suspension ( $50 \mathrm{mg} / 5 \mathrm{ml}$ ) was held between two opposite parallel flat magnetic poles, $20 \mathrm{~mm}$ apart (Figure 1).

The experiments were carried out in a configuration with vectors of magnetic induction perpendicular to the force of gravity. The maximum magnetic induction $B$ available was 1.3 Tesla. Sedimentation was first recorded in the absence, and subsequently in the presence of the magnetic field of 1 Tesla.

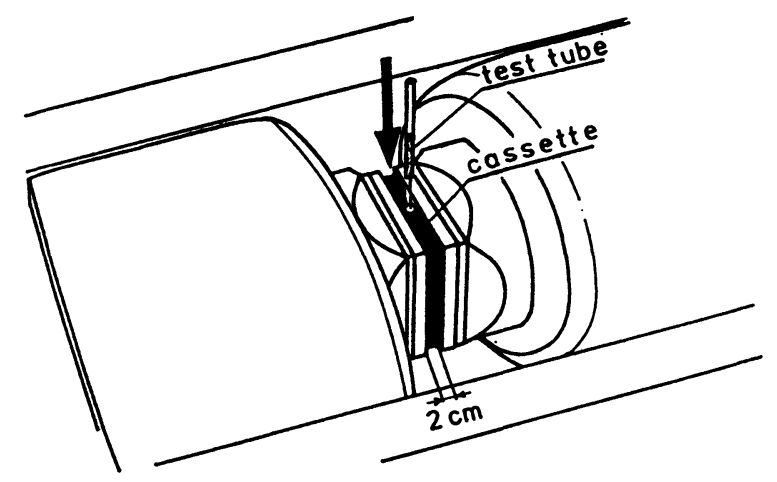

FIGURE 1. Schematic representation of the sedimentation cassette situated between magnetic poles with the test tube inserted.

A useful measure of wettability of siderite is the water contact angle. This parameter was measured on pressed discs of siderite powder, previously conditioned with a surfactant, filtered and dried. 


\section{RESULTS AND DISCUSSION}

\section{Solubility of siderite}

Siderite, a member of the carbonate group of minerals, is hydrophilic and moderately soluble in water. This fact can be documented by measuring $\mathrm{pH}$ of its suspension, as a function of time (Figure 2).

It can be seen from the record that, without the presence of sodium oleate $\mathrm{NaOl}$ (curve a), the initial value of $\mathrm{pH}$ increases from $\mathrm{pH}$ 5.1. to $\mathrm{pH} 7.5$ in five minutes. For comparison, the time change of $\mathrm{pH}$ for siderite suspension in the presence of $\mathrm{NaOl}\left(3 \times 10^{-4} \mathrm{M}\right)$ is shown (curve b).

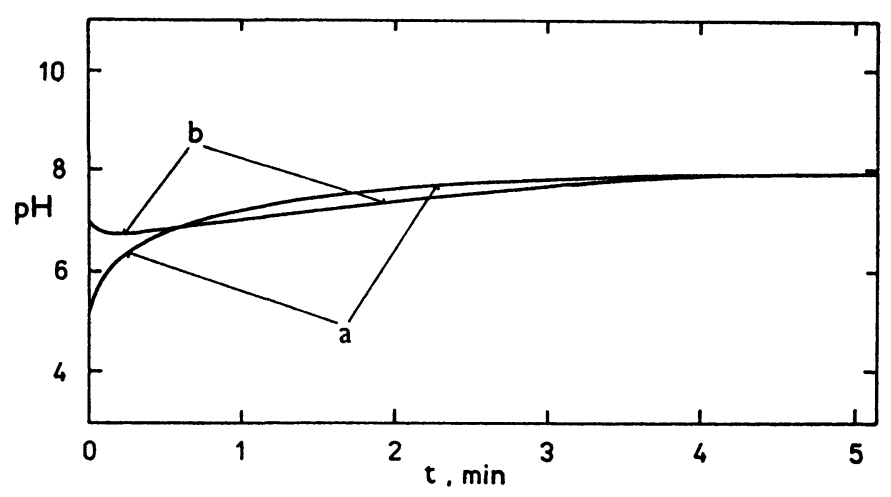

FIGURE 2. The $\mathrm{pH}$ of siderite suspension as a function of time. (a) distilled water, (b) $3 \times 10^{-4} \mathrm{M} \mathrm{NaOl}$.

\section{Wettability of siderite}

Wettability of siderite as a function of $\mathrm{NaOl}$ concentration was evaluated by measuring the water contact angle $\theta_{w}$. The resultant sharp increase in hydrophobicity of siderite is shown in Figure 3.

A similar effect has been observed for magnesite and dolomite suspensions in the presence of $\mathrm{NaOl}$ [2]. Thus, one can believe that the surface of siderite particles has the maximum hydrophobicity to be coated with precipitates of Fe oleate.

The aim of this paper is to explain the fundamentals of magnetic flocculation of paramagnetic minerals in connection with polar interaction forces and the mechanism of adsorption of $\mathrm{NaOl}$ is thus not examined here. 


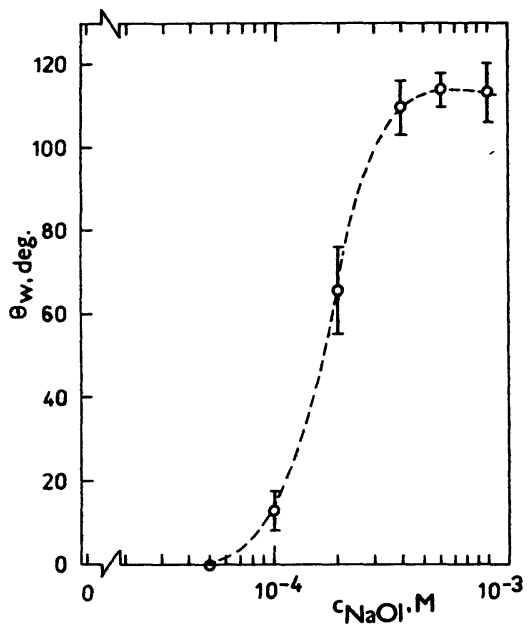

FIGURE 3. Water contact angle of wetting of siderite as a function of $\mathrm{NaOl}$ concentration.

\section{Aggregation of siderite}

Voltage records of sedimentation of siderite suspensions in the presence of different $\mathrm{NaOl}$ concentrations $\left(0\right.$ to $\left.10^{-3} \mathrm{M}\right)$ and at $10^{-2} \mathrm{M} \mathrm{KCl}$ are presented in Figures $4 \mathrm{a}-\mathrm{h}$. It can be seen that the decrease in the turbidity of suspensions during sedimentation is associated with the voltage increase. Three regions can be distinguished in these settling characteristics. The region denoted as $\mathrm{A}$ represents an initial stage of sedimentation above the level of the detecting sensor (see Figure 4a); region B, accompanied by a sudden decrease in the turbidity, starts to take place when the sedimentation interface has reached the sensor, and finally, region $\mathrm{C}$ reflects the final stage where suspensions are almost clear so that the voltage increases with time only negligibly.

For each $\mathrm{NaOl}$ concentration, as shown in Figure 4, the sedimentation record was taken first without the magnetic field and then under the influence of magnetic field of the value of $1 \mathrm{~T}$.

In Figures $5 \mathrm{a}$ and $6 \mathrm{a}$ the voltage changes against the particular $\mathrm{NaO}$ concentrations used are shown for 2 and 7 minute periods of sedimentation, respectively. 

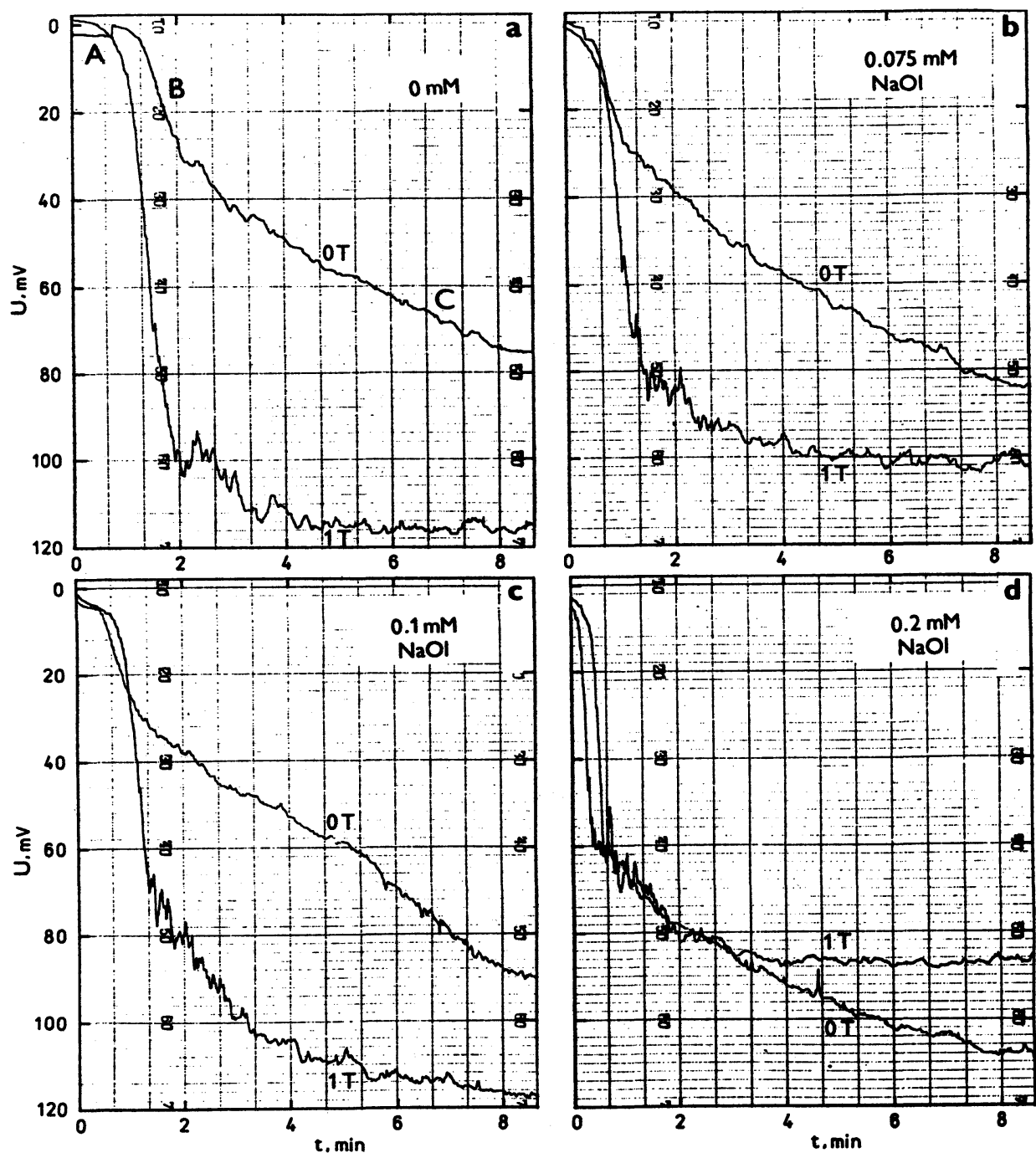

FIGURE 4. Voltage records for sedimentation of siderite, in the presence of $\mathrm{NaOl}$ without and with the magnetic field $(\mathrm{B}=1 \mathrm{~T}), 10^{-2} \mathrm{M}$ $\mathrm{KCl}$.

It follows from the sedimentation patterns, at $10^{-4} \mathrm{M} \mathrm{NaOl}$ and below (Figures 4ac), the sedimentation of siderite, in the absence of the magnetic field, is slow, i.e. the suspension is stable, and that the magnetic field causes a sudden increase in the rate of sedimentation. 

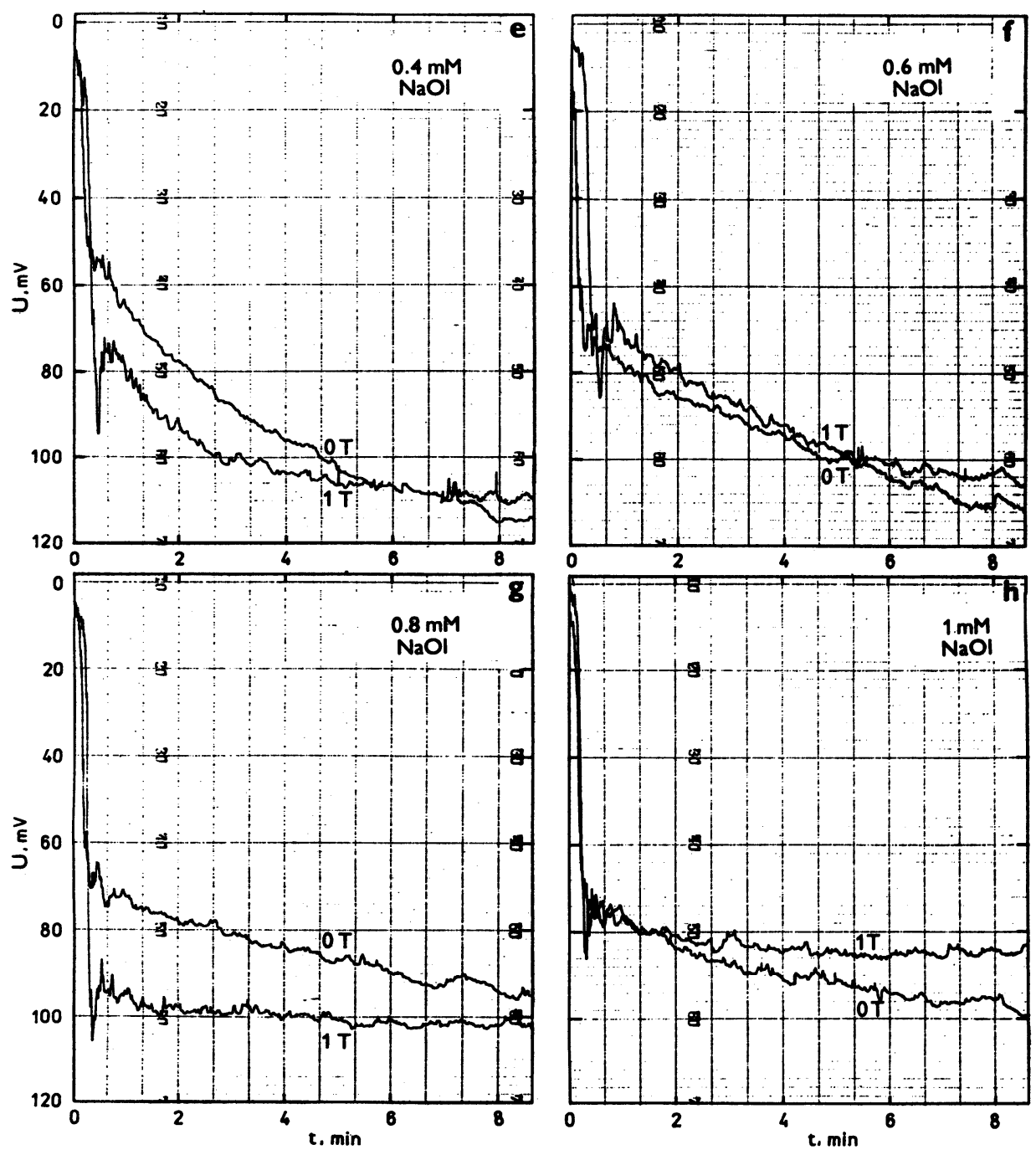

FIGURE 4 Continuation from previous page

However, a further increase in $c_{\mathrm{NaOl}}$ causes a dramatic increase in the sedimentation, even in the absence of the magnetic field (Figures $4 d-h)$. Moreover, the magnetic field does not influence the observed pattern.

Similar records (not shown) have been taken in the absence of $\mathrm{KCl}$. The corresponding voltages, as a function of $c_{\mathrm{NaOl}}$ are given in Figures $5 \mathrm{~b}$ and $6 \mathrm{~b}$ for $\mathrm{t}$ $=2$ and 7 minutes, respectively. It can be seen that under these conditions the observed tendencies are the same as those in the presence of $10^{-2} \mathrm{M} \mathrm{KCl}$, but the 
increase in the sedimentation rate, as a result of the presence of $\mathrm{NaOl}$ in concentration greater than $10^{-4} \mathrm{M}$ is less effective.

In systems with sodium dodecylsulphate (SDS), the sedimentation changes in a somewhat different way. Voltage records for the system are shown in Figures 7a-g. Here, sedimentation steeply decreases and subsequently increases with increasing Concentration of SDS (Figure 8). In comparison with $\mathrm{NaOl}$ the increase is small, but general trends are the same as those observed with previous systems.

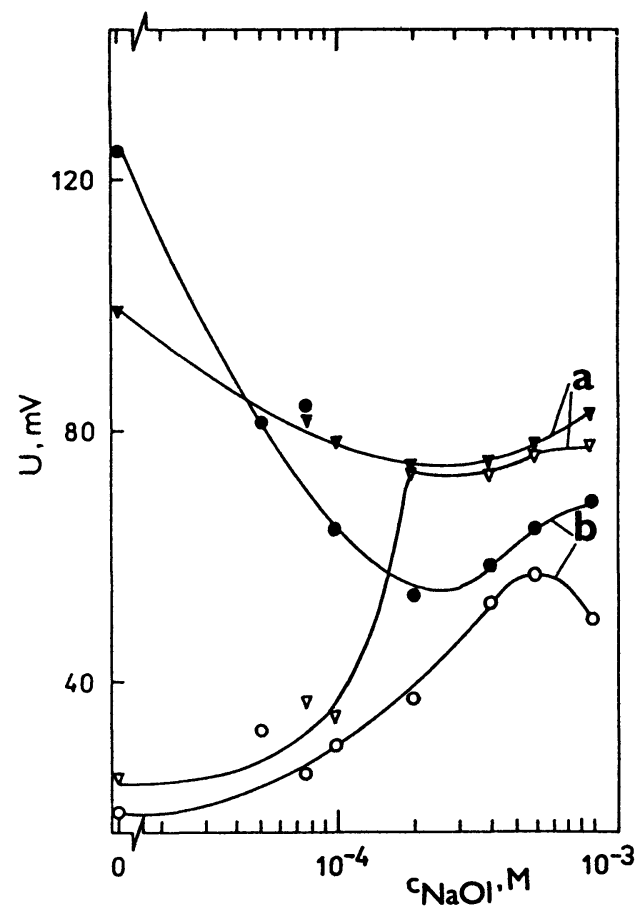

FIGURE 5. Voltage changes after a 2 minute period of sedimentation of siderite at $10^{-2} \mathrm{M}$ (a) and 0 (b) $\mathrm{KCl}$, as a function of concentration of $\mathrm{NaOl}$. Open symbols: $\mathrm{B}=0 \mathrm{~T}$, Closed symbols: $\mathrm{B}=1 \mathrm{~T}$.

\section{Application of the model}

An explanation for the increasing velocity of sedimentation of siderite by the magnetic field at low $\mathrm{c}_{\mathrm{NaOl}}$ is probably aggregation in the secondary minimum. This fact can be documented by Figure 9 where total interaction energies (in KT units) are plotted versus the separation between hydrophilic siderite spheres. These curves were calculated using the equations presented in previous paper [1], assuming $\mathrm{a}=1 \mu \mathrm{m}, \gamma_{\mathrm{SL}}^{\mathrm{AB}}=-20 \mathrm{~mJ} \cdot \mathrm{m}^{-2}, \mathrm{~A}=10^{-20} \mathrm{~J}, \kappa=3.256 \times 10^{8} \mathrm{~m}^{-1}$ and $\mathrm{h}_{\mathrm{cr}}$ 
$=0.2 \mathrm{~nm}$. The polar (acid-base) component of interfacial tension, $-20 \mathrm{~mJ} \cdot \mathrm{m}^{-2}$, was chosen as a representative value for hydrophilic carbonates.

In Figure 9, the upper two curves were obtained for the zeta potential of $50 \mathrm{mV}$ (solid lines) and of $0 \mathrm{mV}$ (dashed lines), assuming no effect of the magnetic field. It can be seen that there should be a strong polar (hydratation) repulsion between the hydrophilic siderite particles, that prevents aggregation.

Actually, the siderite suspensions with $\mathrm{c}_{\mathrm{NaOl}}<10^{-4} \mathrm{M}$ were fully stable in the absence of the magnetic field. On the other hand, the shape of the analogous curves for $B=1 \mathrm{~T}$ (the lower two curves in Figure 9) clearly indicates the feasibility of aggregation of hydrophilic siderite at large separations, i.e. in the secondary minimum.

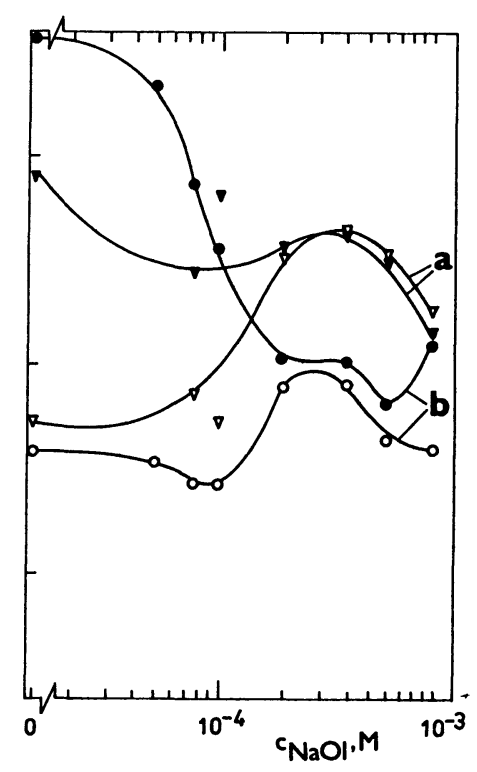

FIGURE 6. Voltage changes after a 7 minute period of sedimentation of siderite. See also the caption of Figure 5.

Again, this assumption was confirmed experimentally. both phenomena are consistent with the assumption that results from Figure 5 in paper [1] obtained for hydrophilic paramagnetic minerals.

When $\mathrm{NaOl}$ is present at higher concentration $\left(>10^{-4} \mathrm{M}\right)$ the increasing sedimentation is assumed to be caused by aggregation in the primary minimum. The argument is that at such a concentration, siderite is hydrophobic (see Figure 
3) so that its $\gamma_{S L}^{A B}$ is positive, which leads to attractive polar force causing a strong interparticle attraction at short distances.

In Figure 10, the interaction energy curves, such as those in Figure 9, are drawn assuming $\gamma_{\mathrm{SL}}^{\mathrm{AB}}=50 \mathrm{~mJ} \cdot \mathrm{m}^{-2}$. It is interesting to note that all curves in this figure terminate at the same point in the attractive region and no energy maxima have developed. this is an explanation of why the magnetic field did not influence the increased sedimentation of hydrophobized siderite.
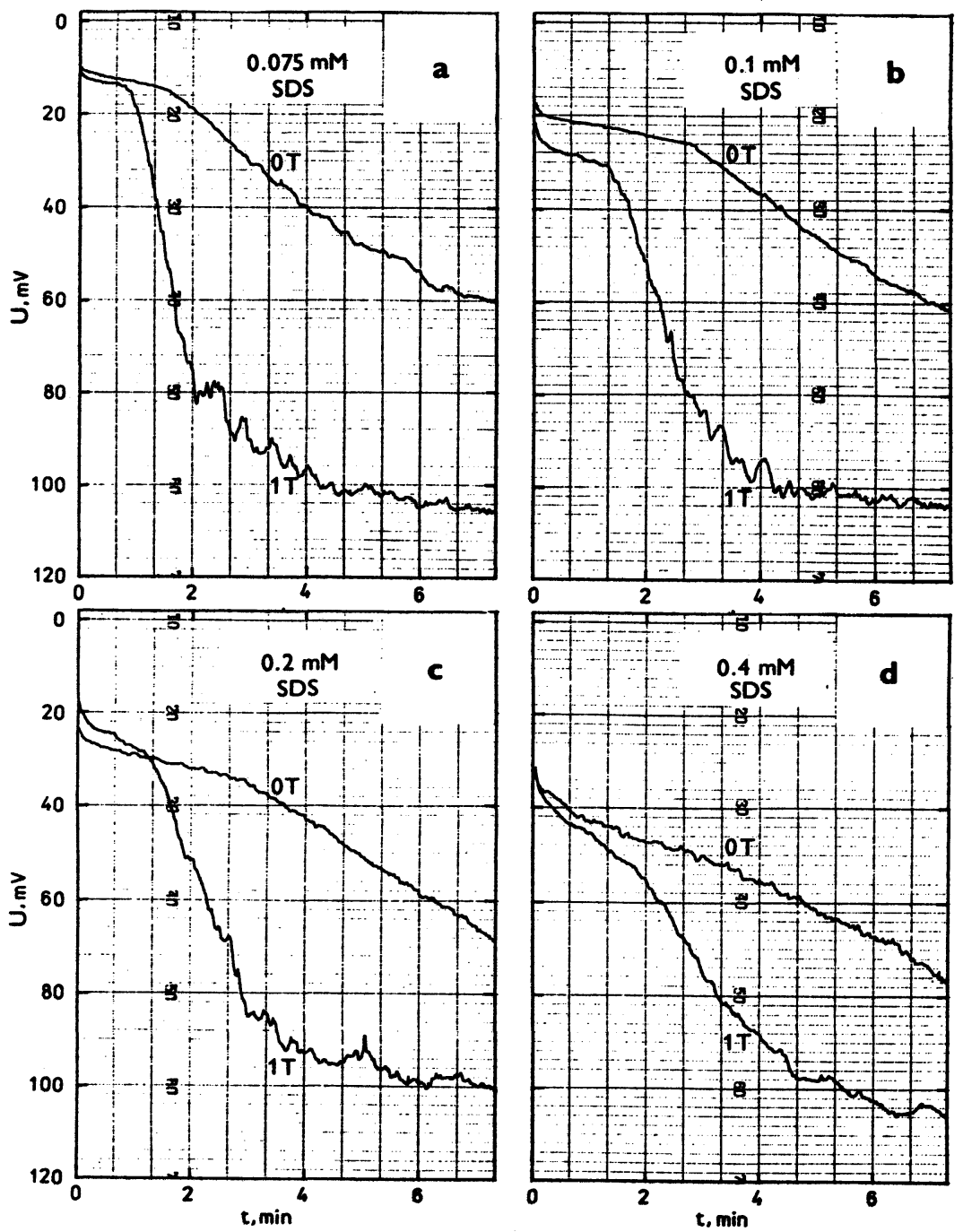

FIGURE 7. Voltage records of sedimentation of siderite in the presence of SDS without and with the magnetic field $(B=1 \mathrm{~T}) \cdot 10^{-2} \mathrm{M}$ $\mathrm{KCl}$. 
In other words, the magnetic dipolar force is only of minor importance when compared with attractive polar forces acting between hydrophobic surfaces of fine paramagnetic minerals. This fact is in accordance with the implication concerning the attractive polar forces in paper [1].
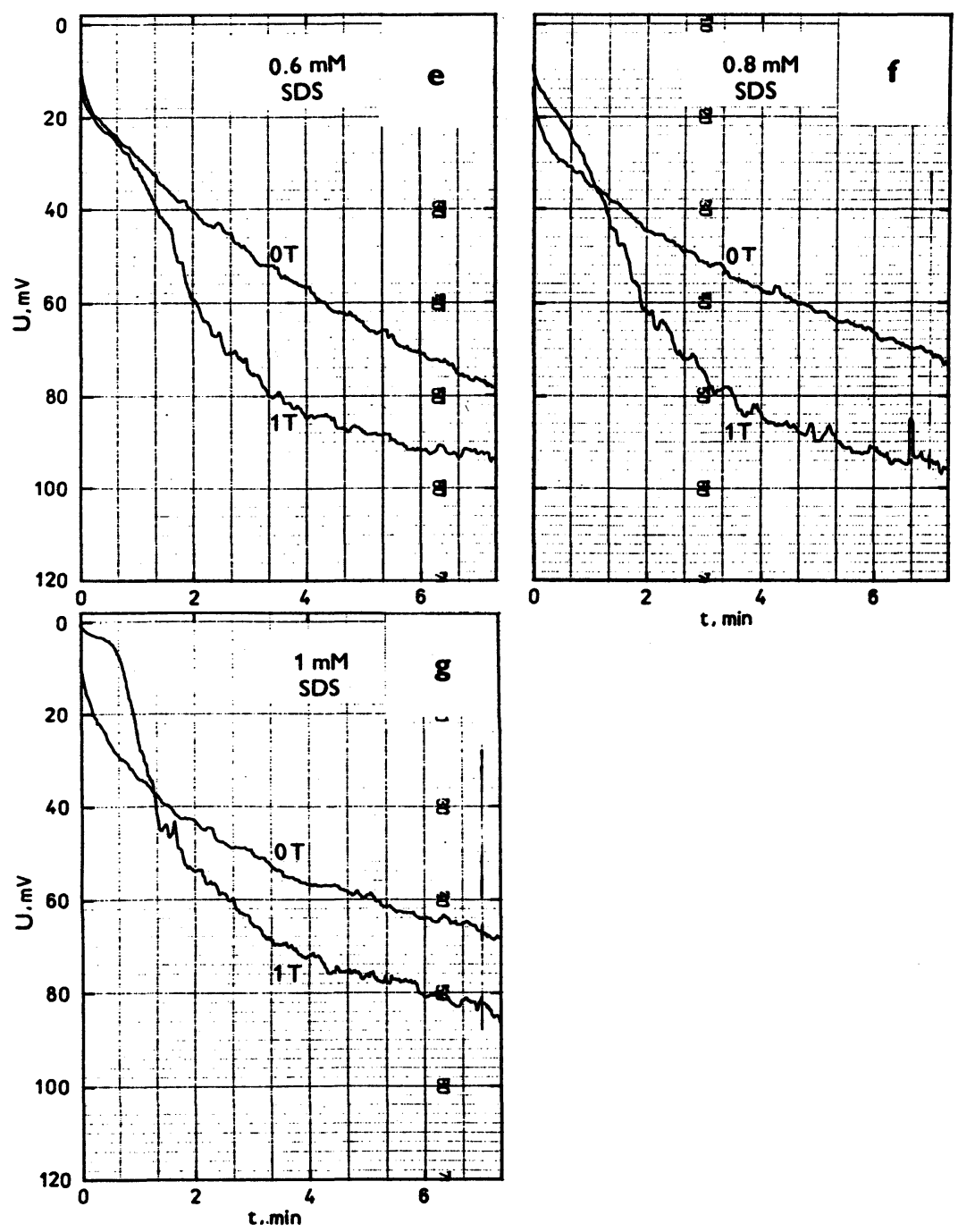

FIGURE 7. Continuation from previous page

The role of electrolyte concentration in aggregation can be explained using the total interaction energy functions calculated for the above hydrophilic (Figure 11) and hydrophobic (Figure 12) siderite particles, assuming $\kappa=10^{8} \mathrm{~m}^{-1}$ . This value of $\kappa$ is chosen to represent a low ionic strength of distilled water and is used to demonstrate the cause of the less effective aggregation of siderite due to 
the attractive polar forces in distilled water (i.e. without $\mathrm{KCl}$, see Figures $5 \mathrm{~b}$ and 6b).

Comparing Figure 11 and Figure 12 with Figure 9 and Figure 10, respectively, one can see that, while in the case of hydrophilic siderite the curve courses differ only slightly (for $B=0$, and for $B=1 \mathrm{~T}$ ), for hydrophobic siderite the energy barrier can be present for higher zeta potentials causing primary aggregation in distilled water to become reduced.

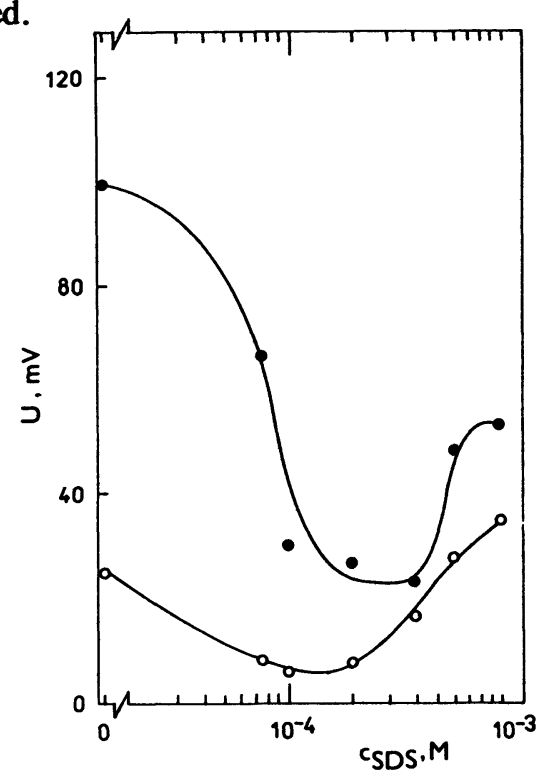

FIGURE 8. Voltage changes after a 2 minute period of sedimentation of siderite at $10^{-2} \mathrm{M}$ as a function of concentration of SDS. Open symbols: $\mathrm{B}=0 \mathrm{~T}$, closed symbols: $\mathrm{B}=1 \mathrm{~T}$.

\section{Character of siderite aggregates}

The proposed conception allows us to estimate the interaction energy between minerals at short distances and in contact, taking into account the polar forces. As shown, two different aggregation patterns have been distinguished theoretically as well as experimentally; namely aggregations in primary and secondary minima.

The former kind of aggregation is valid for interacting hydrophilic siderite as caused by attractive long-range magnetic dipolar force. Primary aggregation is not possible in this case because of the repulsive short-range polar (hydratation) forces that prevent a thermodynamically favourable contact of the particles.

Once the magnetic field is switched off, the secondary minimum vanishes and the full repulsion is restored. These facts result in stability of siderite previously 
aggregated. This phenomenon was actually observed and the photograph (Figure 13a) showing stable hydrophilic siderite particles (without $\mathrm{NaOl}$ ) sedimented under the influence of the magnetic field provides an evidence.
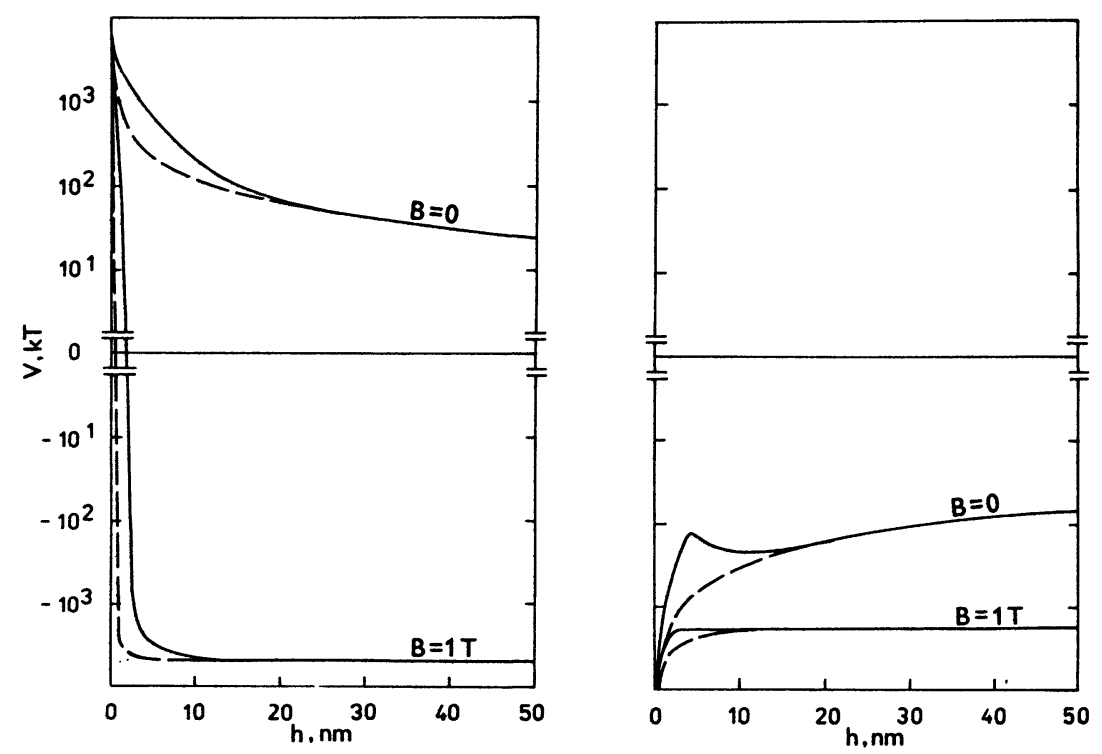

FIGURE 9. Total interaction energies between model hydrophilic siderite spheres at $10^{-2} \mathrm{M} \quad 1-1$ electrolyte. Parameters: $\gamma_{\hat{S} \mathbf{A}}^{\mathbf{B}}=-20$ mJ.m ${ }^{-2}, A=10^{-20} \mathrm{~J}, \kappa=3.256 \times 10^{8} \quad \mathrm{~m}^{-1}, \mathrm{~h}_{\mathrm{cr}}=0.2 \mathrm{~nm}$. Solid lines: $\xi=50 \mathrm{mV}$, dashed lines $\xi=0 \mathrm{mV}$. The curves were calculated using functions presented in previous paper [1].

FIGURE 10. Total interaction energies between model hydrophobic siderite spheres at 10-2 M 1-1 electrolyte. $\gamma_{\mathrm{SL}}^{A B}=50 \mathrm{~mJ} \cdot \mathrm{m}^{-2}$. The remaining parameters are the same as those in Figure 9.

The latter kind of aggregation is a manifestation of interaction of hydrophobic siderite due to the attractive polar (hydrophobic) forces that lead to thermodynamically favourable contact of particles.

These forces result in hydrophobic particles of siderite coming close to one another and the presence of deep primary minimum makes such aggregates difficult to disrupt, irrespective whether the magnetic field is applied or not. A photograph presented in Figure $13 \mathrm{~b}$ shows these aggregates created at $3 \times 10^{-4} \mathrm{M}$ $\mathrm{NaOl}$. 

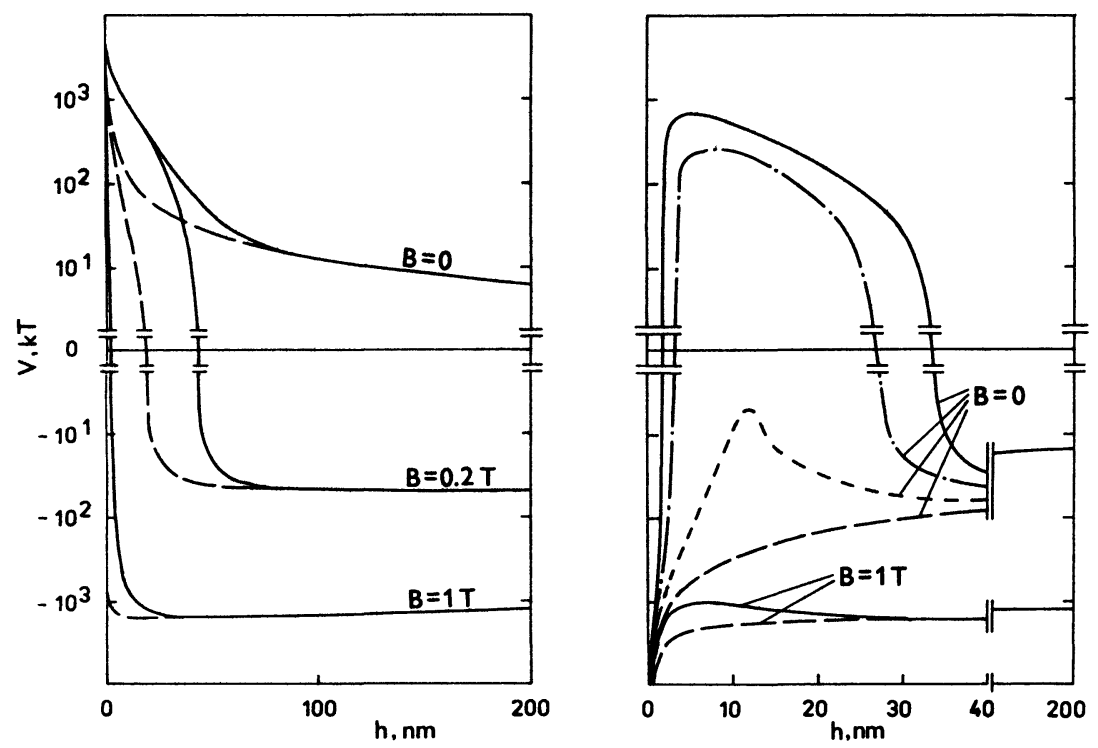

FIGURE 11. Total interaction energies as those in Figure 9, using $\kappa=10^{8}$ $\mathrm{m}^{-1}$ (distilled water).

FIGURE 12. Total interaction energies as those in Figure 10, using $\kappa=10^{8}$ $\mathrm{m}^{-1}$ (distilled water).

\section{CONCLUSION}

This investigation has offered a viable explanation of aggregation of fine paramagnetic minerals as influenced by adsorbed surfactant and by the magnetic field.

A new model of interaction of colloidal particles that takes into account polar forces originating from polar interfacial interactions was applied to the experiments.

Investigation of sedimentation of siderite suspensions in the presence of surfactants shows a close correlation between aggregation of siderite and the nature of functions of the total interaction energy calculated on the basis of the model presented in previous paper [1]. 

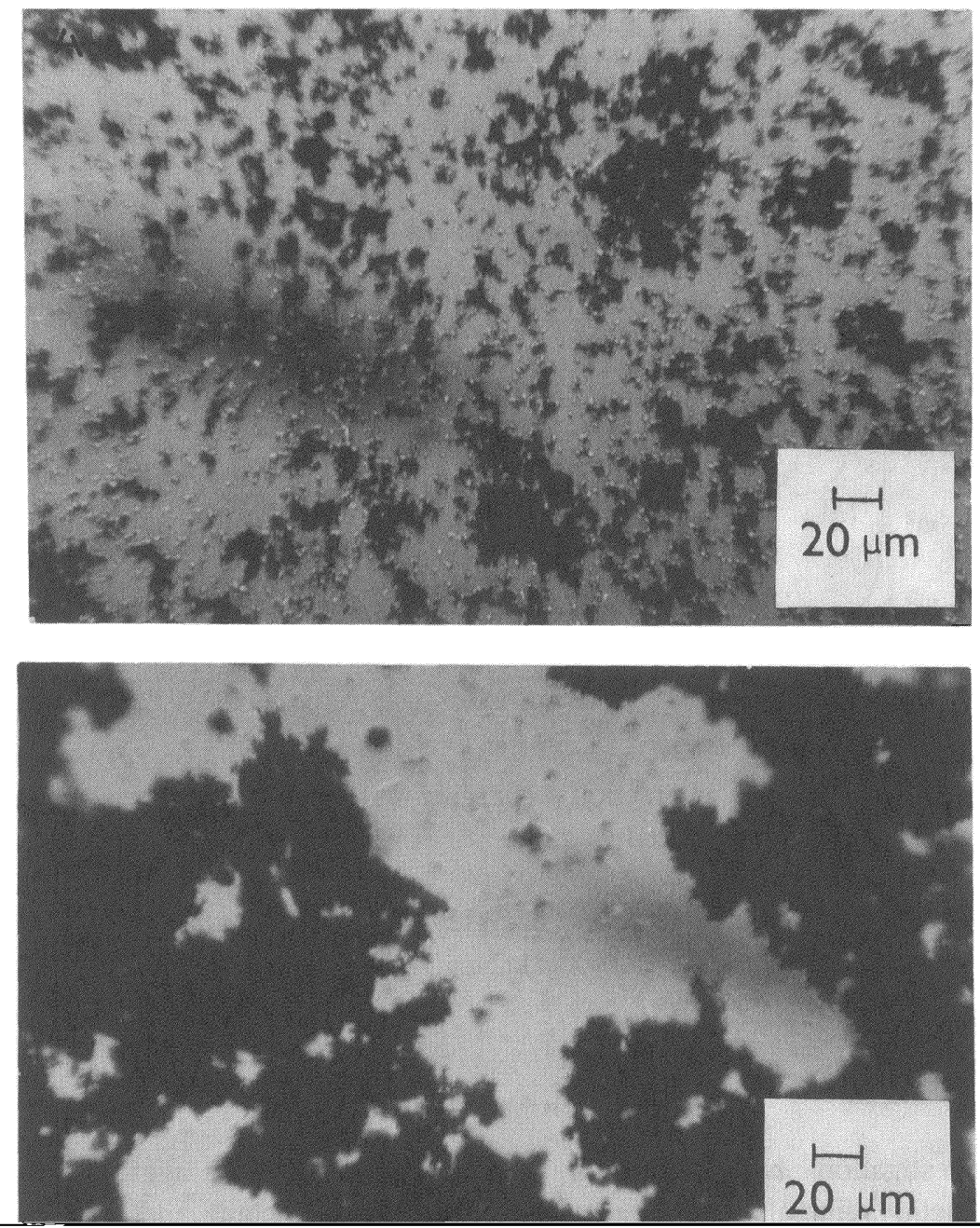